\title{
Well-Posedness of One-Way Wave Equations and Absorbing Boundary Conditions
}

\author{
By Lloyd N. Trefethen* and Laurence Halpern
}

\begin{abstract}
A one-way wave equation is a partial differential equation which, in some approximate sense, behaves like the wave equation in one direction but permits no propagation in the opposite one. Such equations are used in geophysics, in underwater acoustics, and as numerical "absorbing boundary conditions". Their construction can be reduced to the approximation of $\sqrt{1-s^{2}}$ on $[-1,1]$ by a rational function $r(s)=p_{m}(s) / q_{n}(s)$. This paper characterizes those rational functions $r$ for which the corresponding one-way wave equation is well posed, both as a partial differential equation and as an absorbing boundary condition for the wave equation. We find that if $r(s)$ interpolates $\sqrt{1-s^{2}}$ at sufficiently many points in $(-1,1)$, then well-posedness is assured. It follows that absorbing boundary conditions based on Padé approximation are well posed if and only if $(m, n)$ lies in one of two distinct diagonals in the Padé table, the two proposed by Engquist and Majda. Analogous results also hold for one-way wave equations derived by Chebyshev or least-squares approximation.
\end{abstract}

1. Introduction. The wave equation

$$
u_{t t}=u_{x x}+u_{y y}
$$

admits plane wave solutions

$$
u(x, y, t)=e^{i(\tau t+\xi x+\eta y)}
$$

for any $\xi, \eta, \tau \in \mathbf{R}$ that satisfy the dispersion relation

$$
\tau^{2}=\xi^{2}+\eta^{2}
$$

In the $(\xi, \eta)$-plane, the curves of constant $\tau$ determined by this equation are concentric circles. The phase and group velocities of (1.2) are identical and equal to

$$
\mathbf{v}=\left(v_{x}, v_{y}\right)=\left(-\frac{\xi}{\tau},-\frac{\eta}{\tau}\right)=(\cos \theta, \sin \theta), \quad \theta \in[0,2 \pi] .
$$

Thus, for each frequency $\tau,(1.1)$ admits plane waves traveling with speed 1 in all directions.

In certain applications, what is ideally wanted is a one-way wave equation or paraxial equation that admits only half of these solutions: in this paper, all those with $v_{x} \leqslant 0$, i.e., $\theta \in[\pi / 2,3 \pi / 2]$. This idea has been introduced over the years in

Received June 6, 1985; revised February 7, 1986.

1980 Mathematics Subject Classification. Primary 65N99, 65M05, 35L05.

Key words and phrases. Well-posedness, one-way wave equation, paraxial approximation, parabolic approximation, rational approximation, Padé table, absorbing boundary condition, migration, underwater acoustics.

* Research supported by the National Aeronautics and Space Administration under Contract. No. NAS1-17070 while the author was a visitor at ICASE, NASA Langley Research Center, Hampton, VA 23665, and by National Science Foundation Grant DMS-8504703. 
various fields, and some early references are given in the appendix to [27]. In the past decade and a half, one-way wave equations have come into heavy use in three areas. First, they were introduced by Claerbout in 1970 for geophysical migration of seismic waves, in which the aim is to construct images of geological formations underground by downward extrapolation of sound wave reflection data measured at the surface [7]. For subsequent developments see [4], [8], [26] and recent volumes of Geophysics. Second, they were introduced by Tappert and Hardin in 1973 for underwater acoustics calculations, where the simulation of waves in the ocean can be speeded up greatly by assuming one-way lateral propagation away from the source [27]. Further developments in this area are described in [22], [24], the latter of which contains many references, and in recent volumes of the Journal of the Acoustical Society of America. Finally, one-way wave equations were proposed again by Lindman in 1975 [23] and by Engquist and Majda in 1977 [10], [11] for application as approximate absorbing boundary conditions in numerical computations, where artificial boundaries must be introduced to limit a computational domain. For subsequent developments of this and related ideas, see [3], [9], [18] and recent volumes of the Journal of Computational Physics.

Solving for $\xi$ in (1.3), we get

$$
\xi= \pm \tau \sqrt{1-\eta^{2} / \tau^{2}}
$$

where $\sqrt{\cdot}$ denotes the standard branch of the square root. By (1.4), the plus and minus signs correspond to leftgoing and rightgoing waves, respectively:

$$
\begin{gathered}
\xi=+\tau \sqrt{1-\eta^{2} / \tau^{2}} \Leftrightarrow v_{x} \leqslant 0, \\
\xi=-\tau \sqrt{1-\eta^{2} / \tau^{2}} \Leftrightarrow v_{x} \geqslant 0 .
\end{gathered}
$$

Consequently, the ideal one-way equation would be an equation with dispersion relation (1.6a), or equivalently

$$
\xi=\tau \sqrt{1-s^{2}}, \quad s=\eta / \tau=-\sin \theta \quad \text { IDEAL O.W.W.E. }
$$

In the $(\xi, \eta)$-plane, the curves of constant $\tau$ determined by this equation are concentric semicircles. Since $\sqrt{1-s^{2}}$ is not rational, however, (1.7) is not the dispersion relation of a partial differential equation but of an equation containing a pseudodifferential operator. Such an operator is not local in space or time and cannot readily be implemented numerically, especially when the problem is generalized to allow variable coefficients.

To construct practical one-way wave equations, therefore, we will approximate (1.7) with the aid of a function

$$
r(s)=p_{m}(s) / q_{n}(s)
$$

where $p_{m}$ and $q_{n}$ are real polynomials of exact degrees $m \geqslant 0$ and $n \geqslant 0$ with no common zeros. We say that $r$ is a rational function of exact type $(m, n)$. The aim is for $r(s)$ to be a good approximation to $\sqrt{1-s^{2}}$ on $[-1,1]$. From any such approximation, we will derive a corresponding partial differential equation by replacing $(1.7)$ by the dispersion relation

$$
\xi=\tau r(s), \quad s=\eta / \tau=-\sin \theta
$$


For example, suppose $r$ is the Taylor approximant $r(s)=1-\frac{1}{2} s^{2}$. Substituting this for $\sqrt{1-s^{2}}$ in (1.7) gives

$$
\xi=\tau\left(1-\frac{1}{2} \frac{\eta^{2}}{\tau^{2}}\right)
$$

or, equivalently,

$$
\xi \tau=\tau^{2}-\frac{1}{2} \eta^{2}
$$

which is the dispersion relation of

$$
u_{x t}=u_{t t}-\frac{1}{2} u_{y y} .
$$

This is the original one-way wave equation, now classical, and is called the parabolic equation. In the general case (1.8), we multiply both sides by $\tau^{\max \{m-1, n\}} q_{n}(\eta / \tau)$ to clear denominators, and get

$$
\tau^{d-1} \xi q_{n}(\eta / \tau)=\tau^{d} p_{m}(\eta / \tau), \quad d=\max \{m, n+1\}
$$

This equation has the form

$$
P(\xi, \eta, \tau)=0, \quad \operatorname{degree}(P)=d,
$$

where $P$ is a homogeneous polynomial with real coefficients in three variables. Thus it is the dispersion relation or symbol of a homogeneous partial differential equation of order $d$ in $x, y, t$.

The most usual method for obtaining $r$ is by Padé approximation [10], [11]. (Our example above is the Padé approximant of type $(2,0)$.) The disadvantage of Padé approximants is that although they are very accurate for $s \approx 0$, they are inaccurate near the singularities at $s= \pm 1$, and as a result, the corresponding one-way wave equations behave poorly for $\theta \approx \pi / 2,3 \pi / 2$. Therefore, it is tempting to consider alternative choices of $r$. Two reasonable candidates that we treat here are Chebyshev $\left(L_{\infty}\right)$ approximation and least-squares $\left(L_{2}\right)$ approximation. (Certain least-squares approximations for one-way wave equations have been investigated previously [2], [23], [30].) There are many other possibilities too, such as Chebyshev-Padé approximation [12], and we hope to compare the practical merits of various approximation schemes in a later work. (See [33].)

The purpose of this paper is to investigate the well-posedness of one-way wave equations. In one-way wave calculations, as in many other areas of numerical computation, many seemingly reasonable approximations turn out to be ill-posed and hence useless in practice, and it is highly desirable to be able to identify these in advance. Depending on the application, various well-posedness questions arise, of which we will consider two:

Initial value problem (IVP). A one-way wave equation could be applied as a partial differential equation in the domain $t \geqslant 0, x, y \in \mathbf{R}$, subject to initial data at $t=0$. Well-posedness refers to the existence of a unique solution whose norm at $t=t_{0}$ can be estimated in terms of the initial data.

Initial boundary value problem (IBVP). In absorbing boundary condition applications, the domain is $x, t \geqslant 0, y \in \mathbf{R}$, and the one-way wave equation is applied as a boundary condition along $x=0$ for (1.1). Well-posedness is now the existence of a unique solution whose norm at $t=t_{0}$ and along $x=0$ can be estimated in terms of the initial data. 
Our main result is that for most methods of approximation of $\sqrt{1-s^{2}}$, problems IVP and IBVP are well posed if and only if $(m, n)$ lies in one of three diagonals of the table of approximants, or two in the case of even approximants. Our method consists of reducing each well-posedness question to algebraic criteria involving the function $r$, whose relationships to each other are studied systematically. Here is an intuitive explanation of why the result comes out so simply even for the relatively complicated problem IBVP: An absorbing boundary condition permits wave propagation in one direction, while ill-posedness amounts to the possibility of propagation in the other. Thus absorption and well-posedness are naturally related.

According to standard results for hyperbolic partial differential equations, our well-posedness criteria are also valid for one-way wave equations with smoothly varying coefficients [20].

Analogous three-diagonal results have been obtained previously for other problems. In the numerical solution of ordinary differential equations, the Ehle conjecture, proved by Wanner, Hairer, and Nørsett [31], asserts that a certain class of discrete approximations is stable in precisely three diagonals. More recently, Iserles and Strang have established a three-diagonal stability result for discrete approximations to hyperbolic partial differential equations [17]. The connections between these results and ours are at present not understood.

Well-posedness results for one-way wave equations have been proved previously in [1], [2], [9], [11], [30], [32], and elsewhere. In particular, Engquist and Majda showed in [11] that Padé approximation leads to a well-posed problem IBVP for all $m=n$ and $m=n+2$, but an ill-posed one in case $(4,0)$. Thus another way to summarize our results is as follows: We show that the boundary conditions proposed by Engquist and Majda are the only Padé absorbing boundary conditions that are well posed, and we extend an analogous conclusion to other classes of approximations and to initial-value problems.

Two topics must be mentioned that are not discussed in this paper. First, we do not consider the well-posedness of one-way wave equations as evolution equations in $x$ rather than $t$, although this is their most common use in geophysics and underwater acoustics. Second, nothing is said here about discrete approximations to one-way wave equations, which may be unstable even when the differential equation is well posed. We hope to repair these omissions in the future.

2. Statement of Results and Outline of the Paper. Our results can be summarized as follows. Throughout, problems IVP and IBVP are based on the one-way wave equation derived by (1.8) from an arbitrary real rational function $r$ of exact type $(m, n)$ with $r(0) \neq 0, \infty$. Also, the integer $\chi_{m n}$ is defined by

$$
\chi_{m n}= \begin{cases}0 & \text { if } m+n \text { is odd } \\ 1 & \text { if } m+n \text { is even }\end{cases}
$$

THEOREM 1. Problem IVP is well posed if and only if the zeros and poles of $r(s) / s$ are real and simple and interlace along the real axis.

THEOREM 2. Problem IBVP is well posed if and only if the zeros and poles of $r(s) / s$ are real and simple and interlace along the real axis, and furthermore $r(s)>0$ for $s \in[-1,1]$. 
These results imply:

Corollary 1. Problems IVP and IBVP can be well posed only if $n \leqslant m \leqslant n+2$.

COROLlary 2. IBVP well posed $\Rightarrow I V P$ well posed.

Corollary 1 has the following converse:

THEOREM 3. If $n \leqslant m \leqslant n+2$, and if $r(s)$ interpolates $\sqrt{1-s^{2}}$ at $m+n+1+$ $\chi_{m n}$ points in $(-1,1)$, counted with multiplicity, then problems IVP and IBVP are well posed.

The approximants one might consider in practice usually satisfy this interpolation condition. In particular, this is true of Padé, Chebyshev, and least-squares approximants. (The least-squares approximants in question are required to be even, for as we will explain, the situation becomes more complicated otherwise.) This implies finally:

Theorem 4. The Padé, Chebyshev, and least-squares families of one-way wave equation yield well-posed problems IVP and IBVP in precisely two distinct diagonals of the tables of approximants, namely $m=n$ and $m=n+2$.

A remark should be made about odd values of $m$ and $n$. Usually, as in Theorem $4, r$ is an even function, and the resulting one-way wave equation is symmetric about $\theta=\pi$. In this event $m$ and $n$ are even, so the case $m=n+1$ of Theorem 3 does not occur. But there are also applications in which one wants a one-way wave equation tuned asymmetrically to be accurate near some angle $\theta_{0} \neq \pi$, and here, $m$ or $n$ may be odd. We have therefore considered it important to derive results for arbitrary $m$ and $n$ rather than assume these numbers are even.

Here is an outline of the remainder of the paper. Section 3 is devoted to investigating certain purely algebraic properties of $r$ that constitute the heart of our argument. First, Lemma 1 establishes three equivalent statements to the interlace condition of Theorem 1, assuming $r(0)>0$. Thus a fuller assertion than Theorem 1 would be that problem IVP is well posed if and only if $r$ or $-r$ satisfies the conditions of Lemma 1. Next, Lemma 2 establishes three equivalent statements to the condition of Theorem 2; problem IBVP is well posed if and only if $r$ satisfies the conditions of Lemma 2. One of these is an interpolation condition from which Theorem 3 will follow. Finally, Lemma 3 shows that a rational function $r$ can interpolate $\sqrt{1-s^{2}}$ in at most $m+n+1+\chi_{m n}$ points in the plane. This conclusion has been used already in the proof of Lemma 2, and will be applied again later to establish nondegeneracy of Padé and Chebyshev approximants. Because of it, Theorem 3 would be unchanged if it read at least $m+n+1+\chi_{m n}$ points of interpolation.

Section 4 treats well-posedness of problem IVP by reducing it to one of the algebraic conditions of Lemma 1 . This proves Theorem 1 .

Section 5 treats well-posedness of problem IBVP by reducing it to one of the conditions of Lemma 2. This proves Theorems 2 and 3.

Finally, Section 6 shows that various families of even approximants to $\sqrt{1-s^{2}}$, including Padé, Chebyshev, and least-squares, satisfy the interpolation condition of Theorem 3. Together with Corollary 1, this proves Theorem 4. 
3. Lemmas on the Rational Function $r$. Our first lemma identifies a class of functions that decrease monotonically along $\mathbf{R}$ except for simple poles. Parts of this result have appeared elsewhere, for example in [1], [2], [32].

LEMMA 1. Let $r$ be a real rational function of exact type $(m, n)$ with $r(0) \neq 0, \infty$. The following conditions are equivalent:

(a) If $s$ is finite with $\operatorname{Im} s>0$, then $r(s) / s$ is finite with $\operatorname{Im} r(s) / s<0$.

(b) $r(0)>0$, and the zeros and poles of $r(s) / s$ in $\mathrm{C}$ are real and simple and interlace along the real axis.

(c) $r(s) / s$ can be written in the form

$$
\frac{r(s)}{s}=a-b_{0} s+\sum_{k=1}^{n+1} \frac{b_{k}}{s-s_{k}}
$$

for some $a, b_{k}, s_{k} \in \mathbf{R}$, where $s_{1}<s_{2}<\cdots<s_{n+1}, b_{0} \geqslant 0$, and $b_{k}>0$ for $k \geqslant 1$.

Proof. (a) $\Rightarrow$ (b). We will show that if any of the four assertions of condition (b) fails, then (a) cannot hold. First, if $r(0)<0$, then $r(s) / s$ behaves like a positive multiple of $-1 / s$ near $s=0$, and necessarily maps $i \varepsilon$ into the upper half-plane for small enough $\varepsilon$. Second, if $r(s) / s$ has a zero or pole $s_{0} \in \mathbf{C}-\mathbf{R}$, we can assume Im $s_{0}>0$ by symmetry, and $r(s) / s$ must take values in both the upper and lower half-planes near $s_{0}$. Third, if $r(s) / s$ has a multiple zero or pole $s_{0} \in \mathbf{R}$, then $r(s) / s$ must take values in both half-planes for $s$ in a one-sided neighborhood of $s_{0}$ with Im $s>0$. Finally, suppose the zeros and poles are real and simple but do not interlace-there are two zeros $s_{1}, s_{2} \in \mathbf{R}$ with no pole in-between, or the reverse. Then $r(s) / s$ has a local maximum or minimum $\sigma$ at some point $s^{*} \in\left(s_{1}, s_{2}\right)$, so $r(s) / s-\sigma$ has a multiple zero at $s^{*}$, and this is inconsistent with (a) as before.

(b) $\Rightarrow$ (c). Condition (b) implies that $r(s) / s$ has exactly $n+1$ simple poles and at most $n+2$ simple zeros, hence order at most $O(s)$ at $s=\infty$. Therefore its partial fraction decomposition has the form (3.1) for some $a, b_{k}, s_{k} \in \mathbf{R}$ with $s_{1}<\cdots<$ $s_{n+1}$ and with $b_{k} \neq 0$ for $k \geqslant 1$, and if $k_{0}$ is the index with $s_{k_{0}}=0$, then $b_{k_{0}}=r(0)>0$. Now if $b_{k}<0$ for some $k \geqslant 1$, there must be a pair $b_{k}, b_{k+1}$ with $b_{k} b_{k+1}<0$. But in this event there are an even number of zeros between $s_{k}$ and $s_{k+1}$, contradicting the interlace condition. On the other hand, if $b_{k}>0$ for all $k \geqslant 1$ but $b_{0}<0$, then $r(s) / s$ has constant sign and is therefore nonzero on $\left(-\infty, s_{1}\right)$ and $\left(s_{n+1}, \infty\right)$. It follows that if the interlace condition holds, $r(s) / s$ has only $n$ real zeros out of $n+2$ zeros altogether, which implies the existence of a conjugate pair of zeros off the real axis, contradicting (b).

(c) $\Rightarrow$ (a). Trivial.

The conditions of Lemma 1 are enough to ensure that $r(s)$ does not interpolate $-\sqrt{1-s^{2}}$ in the upper half-plane or at $s=0$. For well-posedness of problem IBVP, this will have to be strengthened to exclude interpolation throughout $[-1,1]$. In what follows, $\sqrt{1-s^{2}}$ denotes the branch of the square root defined by branch cuts $(-\infty,-1]$ and $[1, \infty)$ that takes the value 1 at $s=0$.

LEMMA 2. Let $r$ be a real rational function of exact type $(m, n)$ with $r(0) \neq 0, \infty$. The following conditions are equivalent:

(d) $r$ satisfies the conditions of Lemma 1 , and moreover $r(s)>0$ for $s \in[-1,1]$.

(e) $r(s)=-\sqrt{1-s^{2}}$ has no solutions in $\mathbf{C}-(-\infty,-1)-(1, \infty)$. 
(f) $n \leqslant m \leqslant n+2$, and $r(s)=+\sqrt{1-s^{2}}$ has $m+n+1+\chi_{m n}$ solutions in C $-(-\infty,-1]-[1, \infty)$, counted with multiplicity.

Proof. (d) $\Rightarrow$ (e). For $\operatorname{Im} s>0$, we have $\operatorname{Im}\left[-\sqrt{1-s^{2}} / s\right]>0$, while by condition (a) of Lemma $1, \operatorname{Im} r(s) / s<0$. Therefore $r(s)=-\sqrt{1-s^{2}}$ has no solutions in the upper half-plane, or by symmetry, in the lower half-plane. On $[-1,1]$ it cannot have any solutions either, since $-\sqrt{1-s^{2}} \leqslant 0<r(s)$ by assumption.

(e) $\Rightarrow$ (f). Squaring $r(s)= \pm \sqrt{1-s^{2}}$ yields the polynomial equation

$$
p_{m}^{2}(s)-\left(1-s^{2}\right) q_{n}^{2}(s)=0
$$

which has exact degree $2 d=2 \max \{m, n+1\}$, hence has $2 d$ solutions counted with multiplicity, which can only lie in $\mathbf{C}-(-\infty,-1]-[1, \infty)$ since (e) prohibits solutions at $s= \pm 1$. Each of these is a solution of $r(s)=\sqrt{1-s^{2}}$ or of $r(s)=$ $-\sqrt{1-s^{2}}$, but not both, so if (e) holds, there must be $2 d$ solutions of the latter equation. Since $2 \max \{m, n+1\} \geqslant m+n+1+\chi_{m n}$, with equality if and only if $n \leqslant m \leqslant n+2$, condition (f) now follows from Lemma 3 below.

(f) $\Rightarrow$ (d). Condition (f) can hold only if the estimates are sharp in the proof of Lemma 3 below, and this implies the interlace property (b) of Lemma 1; we omit the details. Moreover, $r(s)$ must be positive on $[-1,1]$, for otherwise $r(s)=-\sqrt{1-s^{2}}$ would have a solution there, and for $n \leqslant m \leqslant n+2$, this would put the total number of solutions of (3.2) above the limit $2 \max \{m, n+1\}$.

Finally, Lemma 3, whose proof we have just appealed to, limits the number of times $r(s)$ can interpolate $\sqrt{1-s^{2}}$ in the plane. The following argument is based on ideas suggested to us by Peter Borwein of Dalhousie University. See also Lemma 4.1 of [17], where Rolle's theorem is used to limit the number of interpolation points on an interval. Arieh Iserles has further indicated to us that an alternative proof of Lemma 3 can be based on order stars [17], [31].

LEMMA 3. Let $r$ be a real rational function of exact type $(m, n)$, and let $L$ be the number of solutions of $r(s)=\sqrt{1-s^{2}}$ in $\mathbf{C}-(-\infty,-1)-(1, \infty)$, counted with multiplicity. Then

$$
L \leqslant m+n+1+\chi_{m n} .
$$

Proof. If there is a solution at $s= \pm 1$, its multiplicity is defined to be 1 , since $r(s)$ cannot match the infinite derivative there. But we can dispose of this possibility by considering a new rational function $r_{\varepsilon}(s)=r(s+\varepsilon)$ or $r_{\varepsilon}(s)=r(s+\varepsilon s)$, depending on the signs of $r(s)$ near the endpoints of $[-1,1]$. For $\varepsilon$ sufficiently small and of appropriate sign, $r_{\varepsilon}=\sqrt{1-s^{2}}$ will also have $L$ solutions, all of them in the open region $\mathbf{C}-(-\infty,-1]-[1, \infty)$. So without loss of generality, we can assume $r( \pm 1) \neq 0$.

The number $L$ is the number of zeros of

$$
\phi(s)=p_{m}(s)-\sqrt{1-s^{2}} q_{n}(s)
$$

in $\mathbf{C}-(-\infty,-1]-[1, \infty)$. For sufficiently large $R$, these must all lie in the region shown in Figure 1. Therefore $\phi$ has winding number $L$ along the boundary $\alpha-\beta-\gamma-\bar{\gamma}-\bar{\beta}-\bar{\alpha}$. This implies that $\operatorname{Im} \phi(s)$ and $\operatorname{Re} \phi(s)$ must each have at least $2 L$ zeros interlacing on this boundary. In what follows we assume $m \geqslant n+1$, and bound the number of zeros of $\operatorname{Im} \phi(s)$; for $m \leqslant n+1$, one proceeds analogously by bounding the number of zeros of $\operatorname{Re} \phi(s)$. 


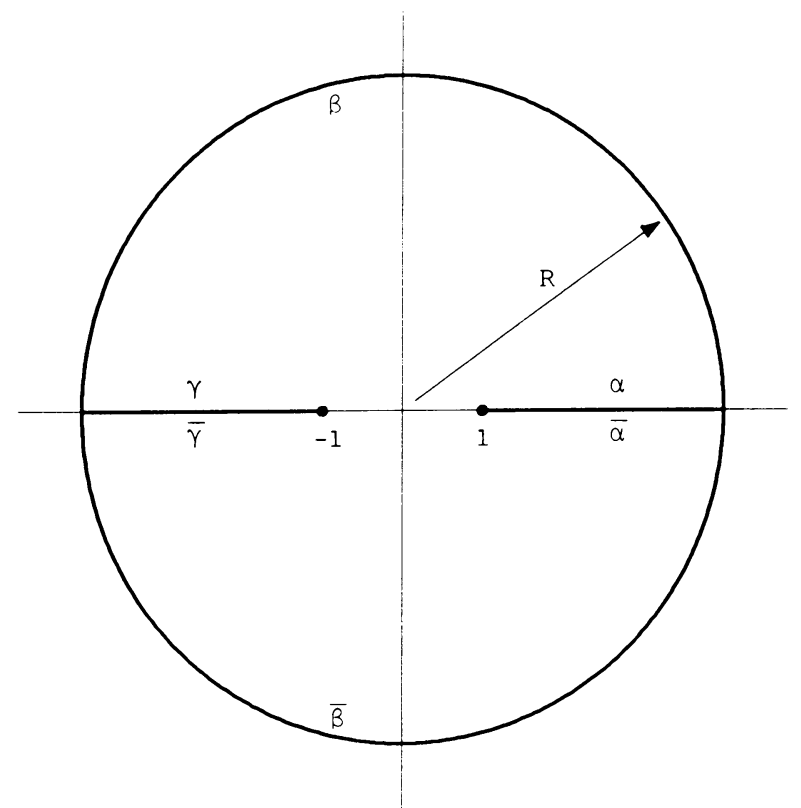

Figure 1

Contour for counting zeros of $r(s)-\sqrt{1-s^{2}}$.

On $\beta, \phi(s)$ behaves approximately like $A s^{m}-i B s^{n+1}$, where $A$ and $B$ are the leading coefficients of $p$ and $q$, respectively. Therefore $\operatorname{Im} \phi(s)$ has at most $m+\chi_{m n}$ zeros on $\beta$, where the $\chi_{m n}$ results from the fact that if $m$ and $n$ are both even or both odd, the lower-order term $i B s^{n+1}$ may bring about one extra crossing of the real axis. On $\bar{\beta}$ the count is the same. On $\alpha, \gamma, \bar{\gamma}$, and $\bar{\alpha}$, we have $\operatorname{Re} \phi(s)=p_{m}(s)$ and $\operatorname{Im} \phi(s)=-i \sqrt{1-s^{2}} q_{n}(s)$, and therefore $\operatorname{Im} \phi(s)$ has at most $2 n+2$ zeros on these contours, including those at $s= \pm 1$. Adding these bounds together gives

$$
2 L \leqslant 2 m+2 \chi_{m n}+2 n+2 \text {, }
$$

which reduces to (3.3).

4. Well-Posedness of Problem IVP. Let $r$ be a real rational function of exact type $(m, n)$, and consider the corresponding homogeneous partial differential equation of degree $d=\max \{m, n+1\}$ obtained from (1.8)-(1.10). Throughout this section, we assume $r(0) \neq 0, \infty$, which is no restriction in practice since $\sqrt{1-s^{2}}=1$ at $s=0$. By (1.9), the condition $r(0) \neq 0$ amounts to the assumption that the coefficient of $\tau^{d}$ in $P(\xi, \eta, \tau)$ does not vanish, so that $t=0$ is not a characteristic surface for the differential equation. Here is the standard result on well-posedness:

Well-Posedness Criterion. Problem IVP is well posed if and only if $P(\xi, \eta, \tau)=0$ has no solutions with $\xi, \eta \in \mathbf{R}$ and $\operatorname{Im} \tau<0$.

A partial differential equation that satisfies this condition is said to be hyperbolic.

For a precise definition of well-posedness and a derivation of this criterion, which is due originally to Gårding, see Section 5.2(b) of [19] or Sections 12.3-12.5 of [15]. The essential idea is Fourier analysis. For suppose problem IVP admits as a solution a mode (1.2) with $\xi, \eta \in \mathbf{R}$ and $\operatorname{Im} \tau<0$. By homogeneity, for any $\alpha>0$, there is another solution with parameters $(\alpha \xi, \alpha \eta, \alpha \tau)$ that grows at the rate $e^{\alpha t|\operatorname{Im} \tau|}$, and as $\alpha$ can be arbitrarily large, the growth up to a fixed time $t_{0}$ cannot be bounded. 
Here is the proof of Theorem 1.

THEOREM 1. Problem IVP is well posed if and only if the zeros and poles of $r(s) / s$ are real and simple and interlace along the real axis.

Proof. Since the zeros of $P(\xi, \eta, \cdot)$ are continuous functions of $\eta$, in the wellposedness criterion it is enough to look for normal modes with $\eta \neq 0$. By (1.9) and (1.10), since $p_{m}$ and $q_{n}$ have no common zeros, an equivalent well-posedness criterion is therefore that if $\xi \in \mathbf{R}$ and $\eta \in \mathbf{R}-\{0\}$, then

$$
\frac{\tau}{\eta} \frac{p_{m}(\eta / \tau)}{q_{n}(\eta / \tau)}=\frac{\xi}{\eta}
$$

has no solutions with $\operatorname{Im} \tau<0$ - or with $\operatorname{Im} \tau>0$, since the solutions come in conjugate pairs. This is further equivalent to the statement that if $r(s) / s \in \mathbf{R}$ for some $s \in \mathbf{C}-\{0\}$, then $s \in \mathbf{R}$. Contrapositively, if $s \in \mathbf{C}-\mathbf{R}$, then $r(s) / s \in \mathbf{C}-$ $\mathbf{R}$. Since $r(s) / s$ is a continuous function of $s$ away from poles, this is equivalent to the statement that $r(s) / s$ maps the upper half-plane either into itself or into the lower half-plane. That is, either $r$ or $-r$ satisfies condition (a) of Lemma 1. By that lemma, this is the same as saying that either $r$ or $-r$ satisfies condition (b), which is the assertion of Theorem 1.

As mentioned earlier, we have actually proved a stronger result: Problem IVP is well posed if and only if $r$ or $-r$ satisfies any of the conditions of Lemma 1.

5. Well-Posedness of Problem IBVP. Again let $r$ be a real rational function of exact type $(m, n)$, and consider now the use of the corresponding one-way wave equation as a boundary condition at $x=0$ for the wave equation (1.1) in the domain $x>0, y \in \mathbf{R}$. For simplicity we continue to assume $r(0) \neq 0, \infty$, as in the last section. To determine well-posedness, we turn to the theory published by Kreiss in 1970 [20], specialized for the wave equation in the interior, and obtain:

Well- Posedness Criterion. Problem IBVP is well posed if and only if $P(\xi, \eta, \tau)$ $=0$ and (1.3) have no mutual solutions $(\xi, \eta, \tau) \neq(0,0,0)$ with $\eta \in \mathbf{R}, \operatorname{Im} \tau<0$, and with $\xi$ belonging to the branch (1.6b).

Since $\tau$ and $\xi$ are now complex, we have to be careful about what is meant by the branch (1.6b). For $\eta \in \mathbf{R}$ and $\operatorname{Im} \tau<0$, it means $\xi=-\sqrt{\tau^{2}-\eta^{2}}$, the analytic function of $\tau$ and $\eta$ obtained by analytic continuation from the values $\xi=-\tau$ for $\eta=0$. These values of $\xi$ satisfy $\operatorname{Im} \xi>0$. For $\tau \in \mathbf{R}, \xi$ is defined by limits in the half-plane $\operatorname{Im} \tau<0$, and satisfies $\operatorname{Im} \xi>0$ if $|\tau|<|\eta|, \xi \in[-1,1]$ if $|\tau| \geqslant|\eta|$.

For the definition of well-posedness and a derivation of this criterion, see [20] and [10], [11]. Again, however, the essential idea is to look for ill-posed normal modes. Suppose problem IBVP admits a solution (1.2) with $\operatorname{Im} \xi \geqslant 0$ and $\operatorname{Im} \tau \leqslant 0$. If $\operatorname{Im} \xi>0$ (decay as $x \rightarrow \infty$ ) but $\operatorname{Im} \tau<0$ (growth as $t \rightarrow \infty$ ), then by homogeneity, as in the last section, unbounded exponential growth as a function of $t$ is possible. If $\operatorname{Im} \tau=\operatorname{Im} \xi=0$ and (1.6b) holds, on the other hand, the solution is a plane wave with $v_{x} \geqslant 0$ that radiates energy from the boundary into the interior, generating weaker but still unbounded growth. See [13] for a presentation of the Kreiss theory from this physical point of view, and [28] for the analogous discussion of stability of finite difference models. 
Here is the proof of Theorem 2.

TheOREM 2. Problem IBVP is well posed if and only if the zeros and poles of $r(s) / s$ are real and simple and interlace along the real axis, and furthermore $r(s)>0$ for $s \in[-1,1]$.

Proof. If there is a normal mode (1.2) with $\tau=0$, then $\xi= \pm i \eta$, and by (1.9), the equation $P(\xi, \eta, \tau)=0$ reduces to

$$
\pm i A \tau^{d-1-n} \eta^{n+1}=B \tau^{d-m} \eta^{m}, \quad d=\max \{m, n+1\}
$$

for nonzero real constants $A$ and $B$. Whether or not $m=n+1$, this implies $\eta=0$ and thus $\xi=0$. Therefore, we can assume $\tau \neq 0$. Now for $\eta \in \mathbf{R}$ and $\operatorname{Im} \tau<0$, the variable $s=\eta / \tau$ lies in $\mathbf{C}-(-\infty, 0)-(0, \infty)$. Taking limits $\operatorname{Im} \tau \rightarrow 0$ with $\tau \rightarrow 0$ amounts to letting $s$ range over all of $\mathbf{C}$, with points on the two sides of the cuts $(-\infty, 0)$ and $(0, \infty)$ viewed as distinct. By $(1.6 \mathrm{~b})$ and $(1.8)-(1.9)$, the well-posedness criterion now reduces to the condition that for $s$ in this region, the equations $\xi=$ $-\tau \sqrt{1-s^{2}}$ and $\xi=\tau r(s)$ are never simultaneously satisfied. That is, the equation $r(s)=-\sqrt{1-s^{2}}$ must have no solutions, where as in Section 3, the square root is defined by branch cuts $(-\infty,-1]$ and $[1, \infty)$ and the value 1 at $s=0$.

On $(-\infty,-1)$ and $(1, \infty), r(s)=-\sqrt{1-s^{2}}$ cannot be satisfied, because the left-hand side is real or infinite while the right-hand side is imaginary, finite, and nonzero. Thus we have reduced the well-posedness criterion to condition (e) of Lemma 2. By that lemma, an equivalent condition is (d), and by Lemma 1(b), this is equivalent to the assertion of Theorem 2 .

Again, we have actually proved a stronger result: Problem IBVP is well posed if and only if $r$ satisfies any of the conditions of Lemma 2. In particular, by condition (e), it is well posed if and only if $r(s)$ interpolates $\sqrt{1-s^{2}}$ at $m+n+1+\chi_{m n}$ points in $\mathbf{C}-(-\infty,-1]-[1, \infty)$. Therefore, a sufficient condition for well-posedness is the existence of $m+n+1+\chi_{m n}$ points of interpolation in $(-1,1)$, and together with Corollary 2 , this proves Theorem 3 .

If one or two points of interpolation lie at \pm 1 instead of in $(-1,1)$, problem IBVP is ill posed. This is a borderline case of weak ill-posedness, corresponding to a wave (1.2) that propagates tangentially to the boundary $x=0$.

6. Families of One-Way Wave Equations. In this final section we will examine various families of approximants $r(s)$ to $\sqrt{1-s^{2}}$ on $[-1,1]$, which correspond to various families of one-way wave equations. By Theorem 3, well-posedness is assured for $n \leqslant m \leqslant n+2$ if $r(s)$ interpolates $\sqrt{1-s^{2}}$ at $m+n+1+\chi_{m n}$ points of $(-1,1)$, and conversely, the approximants one might consider in practice will almost always satisfy this condition. In fact, different methods of approximation of $\sqrt{1-s^{2}}$ can fruitfully be interpreted and compared as different strategies for the allocation of interpolation points in $(-1,1)$ - hence of angles in $(\pi / 2,3 \pi / 2)$ at which the ideal and approximate one-way dispersion relations coincide.

By a simple change of variables, an even approximation $r(s)$ to $\sqrt{1-s^{2}}$ on $[-1,1]$ is equivalent to an approximation of the same type to the function $|s|$. The approximation of $|s|$ is a well-studied problem in approximation theory, which features the discovery by D. J. Newman in 1964 that whereas type $(n, 0)$ approximants have $L^{\infty}$ error $O(1 / n)$ as $n \rightarrow \infty$, type $(n, n)$ approximants have much 
smaller errors $O\left(\right.$ const $\left.^{-\sqrt{n}}\right)$ [25]. Thus, fortunately, our results show that well-posedness correlates with accuracy as $n \rightarrow \infty$.

Until the end of the section, $r$ is an even function. First, we will show that $\sqrt{1-s^{2}}$ can be interpolated in an arbitrary set of points symmetrically distributed in $(-1,1)$. Then we will consider Chebyshev, Padé, and least-squares approximation, and show that each leads to the appropriate number of interpolation points, thereby proving Theorem 4. (These methods of approximation are illustrative; we make no claim about what method may be best in practice.) In Chebyshev and Padé approximation, uniqueness implies that $r(s)$ is automatically even, for otherwise, $r(-s)$ would be a distinct and equally good approximation. In least-squares approximation, uniqueness does not hold, so we have to impose evenness of $r$ as an explicit constraint.

Symmetric interpolation in arbitrary points. The following construction was pointed out to us by Philip Roe, but was used earlier by Newman [25]. For any even $K \geqslant 2$, let $\pm s_{1}, \ldots, \pm s_{K / 2}$ be a set of $K$ points in $(-1,1)$, counted with multiplicity, except that one of these pairs may be \pm 1 . Set $m=2\lfloor K / 4\rfloor$ and $n=2[K / 4\rceil-2$, so that $m=n$ or $m=n+2$ and $K=m+n+2$. Let $p$ be a nonzero polynomial of degree $K / 2$ that is zero at $\sqrt{1-s_{k}^{2}}$ for each $k$, and set

$$
r(s)=\frac{p(t)+p(-t)}{(-p(t)+p(-t)) / t},
$$

where $t=\sqrt{1-s^{2}}$. Since the numerator is even as a function of $t$, it is a polynomial in $s$ of degree $m$, and since the denominator is even as a function of $t$, it is a polynomial in $s$ of degree $n$. Also, since $|p(-t)|>|p(t)|$ for $t>0$ and $p^{\prime}(0) \neq 0$, $r(s)$ can have no poles or zeros in $[-1,1]$ (we exclude the trivial case $K=2, s_{k}=1$, $r(s) \equiv 0)$. Thus $r(s)=\sqrt{1-s^{2}}$ is equivalent to

$$
p(t)+p(-t)=-p(t)+p(-t)
$$

that is, $p(t)=0$. In other words, (6.1) interpolates $\sqrt{1-s^{2}}$ at the points $\pm s_{k}$, and by Theorem 3 , the corresponding one-way wave equation is well posed.

An alternative and more transparent presentation of this construction is implicit in Section 9 of a paper by Higdon [14]. If we write $\sqrt{1-s_{k}^{2}}=-\cos \theta_{k}$, then $p(\xi / \tau)=0$ is equivalent to

$$
\prod_{k=1}^{K / 2}\left(\xi+\cos \theta_{k} \tau\right)=0
$$

which corresponds to the partial differential equation

$$
\prod_{k=1}^{K / 2}\left(\partial_{x}+\cos \theta_{k} \partial_{t}\right) u=0
$$

which is satisfied exactly by plane waves at the angles $\pm \theta_{k}$. Conversely, given a one-way wave equation in the form (6.3), let us use (1.3) to eliminate all powers of $\xi$ higher than 1 in its dispersion relation (6.2). The result is a new dispersion relation that interpolates $\sqrt{1-s^{2}}$ in the points $s_{k}$ and is equivalent to a rational expression (1.8). For example, Higdon points out that the Engquist-Majda absorbing boundary conditions can be generated in this fashion from $\left(\partial_{x}-\partial_{t}\right)^{K / 2} u=0$, an observation that highlights their close relation to the boundary conditions of Bayliss and Turkel (see Section 2 of [3]). 
Now we turn to Chebyshev, Padé, and least-squares approximation. In what follows, let $r$ denote a real rational function of type $(M, N)$ but not necessarily exact type $(M, N)$; that is, $r$ has exact type $(m, n)$ for some $m \leqslant M, n \leqslant N$. Assume further, until further notice, that $M, N, m$, and $n$ are even integers and that $r$ is an even function. The defect of $r$ (as a function of type $(M, N)$ ) is the integer

$$
\delta=\min \{M-m, N-n\} \geqslant 0 .
$$

Also, let $K$ be the number of zeros of $\sqrt{1-s^{2}}-r(s)$ in $(-1,1)$. By definition $K \leqslant L$, where $L$ is the integer of Lemma 3 , and so by that lemma, since $\chi_{m n}=1$ when $m$ and $n$ are both even, we have

$$
K \leqslant m+n+2 .
$$

Chebyshev approximation. The Chebyshev approximant of type $(M, N)$ to $\sqrt{1-s^{2}}$, denoted $r_{M N}^{*}(s)$, is defined by the condition

$$
\left\|\sqrt{1-s^{2}}-r_{M N}^{*}(s)\right\|_{\infty}=\text { minimum, }
$$

where $\|\cdot\|_{\infty}$ is the supremum norm on $[-1,1]$. It is well known that $r_{M N}^{*}$ exists, is unique, and is characterized by the following "equioscillation condition" [5], [27]. Let $r$ be a rational function of type $(M, N)$, exact type $(m, n)$, and defect $\delta$. Then $r=r_{M N}^{*}$ if and only if $\sqrt{1-s^{2}}-r(s)$ attains alternating values $\pm\left\|\sqrt{1-s^{2}}-r(s)\right\|_{\infty}$ at some sequence of points $-1<s_{0}<\cdots<s_{J} \leqslant 1$ with

$$
J \geqslant M+N+1-\delta .
$$

Let $r=r_{M N}^{*}$ be the Chebyshev approximant to $\sqrt{1-s^{2}}$ of type $(M, N)$. Between any two equioscillation points there must be a zero, so we have $K \geqslant J$, and by (6.6),

$$
K \geqslant M+N+1-\delta .
$$

Together with (6.5), this yields

$$
M+N+1-\delta \leqslant m+n+2
$$

or by (6.4),

$$
M+N+1-\delta \leqslant(M-\delta)+(N-\delta)+2 .
$$

Therefore $\delta \leqslant 1$. Since $\delta$ is even, however, we must have $\delta=0$. Since $K$ is even also, (6.5) and (6.7) now yield

$$
m=M, \quad n=N, \quad K=m+n+2 .
$$

(That is, the Chebyshev table for $\sqrt{1-s^{2}}$ is normal except for the even-odd degeneracy: It breaks into distinct $2 \times 2$ blocks of identical entries $r_{m, n}^{*}=r_{m+1, n}^{*}=$ $r_{m, n+1}^{*}=r_{m+1, n+1}^{*}$.) This establishes the hypotheses of Theorem 3, proving Theorem 4 for Chebyshev approximation.

Padé approximation. The Padé approximant of type $(M, N)$ to $\sqrt{1-s^{2}}$, denoted $r_{M N}^{p}$, is defined by the condition

$$
\sqrt{1-s^{2}}-r_{M N}^{p}(s)=O\left(s^{\text {maximum }}\right) \text { as } s \rightarrow 0 .
$$

Again one has existence and uniqueness (the former is trivial). For this particular case, $r_{M N}^{p}$ is known explicitly and can be constructed from a continued fraction 
expansion [4], [11], [31], from hypergeometric function identities [16], or from (6.1). Alternatively, to emphasize the analogy to Chebyshev approximation, one can reason by means of the following equioscillation-type characterization [29]. Let $r$ be a rational function of type $(M, N)$, exact type $(m, n)$, and defect $\delta$. Then $r=r_{M_{N}}$ if and only if

$$
\sqrt{1-s^{2}}-r(s)=O\left(s^{J}\right) \quad \text { as } s \rightarrow 0
$$

for some $J$ satisfying (6.6). Since (6.9) asserts that $r(s)$ interpolates $\sqrt{1-s^{2}}$ at least $J$ times at the origin, we again have $K \geqslant J$, and the argument leading to (6.8) goes through exactly as before. (That is, the Padé table for $\sqrt{1-s^{2}}$ is also normal except for even-odd degeneracy.) This proves Theorem 4 for Padé approximation.

Least-squares approximation. Rational least-squares approximation, unlike its polynomial counterpart, has certain troublesome properties-notably nonuniqueness and the possibility of local best approximations that are not global. To begin with, let us drop the assumption that $M, N, m$, and $n$ are even. It is known that a least-squares approximant of type $(M, N)$ to $\sqrt{1-s^{2}}$ exists, so let us denote such a function by $r_{M N}^{(2)}$. Here is the remarkable property that distinguishes this problem from Chebyshev and Padé approximation, proved by Cheney and Goldstein in 1967 [6]: $r_{M N}^{(2)}$ always has $\delta=0$. As a corollary, first pointed out by Lamprecht [21], $r_{M N}^{(2)}$ cannot be even when $M$ and $N$ are odd, and so it cannot be unique. We do not know whether it is unique when $M$ and $N$ are even.

Nevertheless, certain conclusions about $r_{M N}^{(2)}$ can be reached despite its lack of uniqueness. Since $\delta=0$, one can show that $\sqrt{1-s^{2}}-r_{M N}^{(2)}$ must be orthogonal on $[-1,1]$ to all polynomials of degree $M+N+1$ and, accordingly, must have $K \geqslant M$ $+N+1$ zeros in $(-1,1)$. (Cheney and Goldstein point this out as their final corollary.) Since $K \leqslant m+n+1+\chi_{m n}$ by Lemma 3, we now reason as follows: If $M$ and $N$ are both even or both odd, then $m=M$ and $n=N$, but we are assured of only $m+n+1$ interpolation points, not the $m+n+2$ required by Lemma 3. On the other hand, if one of $M$ and $N$ is even and the other is odd, then the possibilities are $m=M$ and $n=N, m=M-1$ and $n=N$, or $m=M$ and $n=N-1$, and in each case, $m+n+1+\chi_{m n}=M+N+1$, which guarantees a sufficient number of interpolation points.

We conclude that if one-way wave equations are derived by general least-squares approximation, problems IVP and IBVP are well posed if $M=N+1$, but whether they are well posed for $M=N$ and $M=N+2$ is uncertain.

Symmetric least-squares approximation. The situation returns to normal if, rather than permitting an arbitrary approximant of type $(M, N)$, we require $r$ to be even. (Formula (6.1) will now be a computational help, for we can take the interpolation points as independent variables and vary them systematically to minimize $\left\|\sqrt{1-s^{2}}-r(s)\right\|_{2}$.) Assume again that $M, N, m, n$, and $K$ are even. The problem is equivalent to that of approximating $\sqrt{1-\sigma}$ on $[0,1]$ by a rational function of type $(M / 2, N / 2)$ in the least-squares sense with weight function $\sigma^{-1 / 2}$. By an adaptation of the Cheney-Goldstein argument, we get $\delta=0$ again, from which (6.8) follows. This completes the proof of Theorem 4. 
Acknowledgments. Several of the ideas of Section 3 came to us from Peter Borwein of Dalhousie University, whose assistance is gratefully acknowledged. For additional advice we also thank Robert Almgren, Tony Chan, Robert Clayton, Robert Higdon, Arieh Iserles, and Philip Roe.

Note Added in Proof. Coefficients for several families of one-way wave equations are given in [33].

Department of Mathematics

Massachusetts Institute of Technology

Cambridge, Massachusetts 02139

Centre de Mathématiques Appliquées

Ecole Polytechnique

91128 Palaiseau Cedex, France

1. A. BAMBerger et Al., "The paraxial approximation for the wave equation: some new results," in Advances in Computer Methods for Partial Differential Equations V (R. Vichnevetsky and R. S. Stepleman, eds.), IMACS, 1984.

2. A. Bamberger et al., Construction et Analyse d'Approximations Paraxiales en Milieu Hétérogène I and II, Rapps. Internes Nos. 114 and 128, Centre de Mathématiques Appliquées, Ecole Polytechnique, 1984-85.

3. A. Bayliss \& E. Turkel, "Radiation boundary conditions for wave-like equations," Comm. Pure Appl. Math., v. 33, 1980, pp. 707-725.

4. A. J. BERKHOUT, "Wave field extrapolation techniques in seismic migration, a tutorial," Geophysics, v. 46,1981 , pp. $1638-1656$.

5. E. W. CHENEY, Introduction to Approximation Theory, McGraw-Hill, New York, 1966.

6. E.W. Cheney \& A. A. Goldstein, "Mean-square approximation by generalized rational functions," Math. Z., v. 95, 1967, pp. 232-241.

7. J. F. Claerbout, Imaging the Earth's Interior, Blackwell Publ. Co., 1985.

8. R. W. Clayton, Wavefield Inversion Methods for Refraction and Reflection Data, Ph.D. diss., Dept. of Geophysics, Stanford University, 1981.

9. R. W. Clayton \& B. ENGQUist, "Absorbing boundary conditions for wave-equation migration," Geophysics, v. 45, 1980, pp. 895-904.

10. B. ENGQUist \& A. MAJDA, "Absorbing boundary conditions for the numerical simulation of waves," Math. Comp., v. 31, 1977, pp. 629-651.

11. B. ENGQuist \& A. MAJDA, "Radiation boundary conditions for acoustic and elastic wave calculations," Comm. Pure Appl. Math., v. 32, 1979, pp. 313-357.

12. W. B. Gragg \& G. D. Johnson, "The Laurent-Padé table," in Information Processing 74, North-Holland, Amsterdam, 1974, pp. 632-637.

13. R. L. HIGDON, "Initial-boundary value problems for linear hyperbolic systems," SIAM Rev., v. 28 , 1986, pp. 177-217.

14. R. L. HIGDON, "Absorbing boundary conditions for difference approximations to the multi-dimensional wave equation," Math. Comp., v. 47, 1986, pp. 437-459.

15. L. Hörmander, The Analysis of Linear Partial Differential Operators. II, Springer-Verlag, Berlin and New York, 1983.

16. A. ISERLES, "A note on Padé approximation and generalized hypergeometric functions," BIT, v. 19 , 1979 , pp. $543-545$.

17. A. Iseries \& G. Strang, "The optimal accuracy of difference schemes," Trans. Amer. Math. Soc., v. 277,1983 , pp. $779-803$.

18. M. ISRAELI \& S. A. OrsZAG, "Approximation of radiation boundary conditions," J. Comput. Phys., v. 41,1981 , pp. $115-135$.

19. F. John, Partial Differential Equations, 3rd ed., Springer-Verlag, Berlin and New York, 1978.

20. H.- O. Kreiss, "Initial boundary value problems for hyperbolic systems," Comm. Pure Appl. Math., v. 23, 1970, pp. 277-298.

21. G. LAMPRECHT, "Zur Mehrdeutigkeit bei der Approximation in $\operatorname{der} L^{p}$-Norm mit Hilfe rationaler Funktionen," Computing, v. 5, 1970, pp. 349-355.

22. D. LeE, The State-of-the-Art Parabolic Equation Approximation as Applied to Underwater Acoustic Propagation with Discussions on Intensive Computations, Tech. Doc. 7247, U.S. Naval Underwater Systems Center, New London, Conn., 1984. 
23. E. L. Lindman, “'Free-space' boundary conditions for the time dependent wave equation," $J$. Comput. Phys., v. 18, 1975, pp. 66-78.

24. S. T. MCDANiEL, "Parabolic approximations for underwater sound propagation," J. Acoust. Soc. Amer., v. 58, 1975, pp. 1178-1185.

25. D. J. Newman, “Rational approximation to $|x|$," Michigan Math. J., v. 11, 1964, pp. 11-14.

26. R. H. Stolt, "Migration by Fourier transform," Geophysics, v. 43, 1978, pp. 23-48.

27. F. D. TAPPERT, "The parabolic approximation method," in Wave Propagation and Underwater Acoustics (J. B. Keller and J. S. Papadakis, eds.), Lecture Notes in Phys., vol. 70, Springer-Verlag, Berlin and New York, 1977.

28. L. N. TREFETHEN, "Instability of difference models for hyperbolic initial boundary value problems," Comm. Pure Appl. Math., v. 37, 1984, pp. 329-367.

29. L. N. Trefethen, "Square blocks and equioscillation in the Padé, Walsh, and CF tables," in Rational Approximation and Interpolation (P. R. Graves-Morris et al., eds.), Lecture Notes in Math., vol. 1105, Springer-Verlag, Berlin and New York, 1984.

30. L. WAGATHA, "Approximation of pseudodifferential operators in absorbing boundary conditions for hyperbolic equations," Numer. Math., v. 42, 1983, pp. 51-64.

31. G. Wanner, E. Hairer \& S. P. Nørsett, “Order stars and stability theorems," BIT, v. 18, 1978, pp. 478-489.

32. Zhang GuAN-QUAN, "High-order approximation of one way wave equations," J. Comput. Math., v. 3, 1985, pp. 90-97.

33. L. HAlPERn \& L. N. TRefethen, “Wide-angle one-way wave equations.” (To appear.) 